OPEN ACCESS

Edited by:

Christophe Morisseau, University of California,

Davis, United States

Reviewed by: Mojca Bozic Mijovski, University Medical Centre Ljubljana, Slovenia

Weicang Wang, University of California, Davis, United States

*Correspondence: Yongquan Gu guyqvip@126.com

Specialty section: This article was submitted to Vascular Physiology, a section of the journal Frontiers in Physiology

Received: 20 May 2021 Accepted: 29 July 2021 Published: 24 August 2021

Citation:

Zhou M, Qi L and Gu Y (2021) GRIA2/ENPP3 Regulates the Proliferation and Migration of Vascular Smooth Muscle Cells in the Restenosis Process Post-PTA in Lower Extremity Arteries.

Front. Physiol. 12:712400. doi: 10.3389/fphys.2021.712400

\section{GRIA2/ENPP3 Regulates the Proliferation and Migration of Vascular Smooth Muscle Cells in the Restenosis Process Post-PTA in Lower Extremity Arteries}

\author{
Mi Zhou, Lixing Qi and Yongquan Gu* \\ Department of Vascular Surgery, Xuanwu Hospital, Capital Medical University, Beijing, China
}

Restenosis is the main restriction on the long-term efficacy of percutaneous transluminal angioplasty (PTA) therapy for peripheral artery disease (PAD). Interventions to prevent restenosis are poor, and the exact mechanism is unclear. Here, we aimed to elucidate the role of GRIA2 in the restenosis process post-PTA in lower extremity arteries. We searched the differentially expressed genes (DEGs) between atherosclerotic and restenotic artery plaques in the Gene Expression Omnibus (GEO), and five DEGs were identified. Combined with Gene Ontology (GO) enrichment analysis, GRIA2 was significantly correlated with the restenosis process. Tissue samples were used to examine GRIA2 expression by immunofluorescence staining of atherosclerotic and restenotic artery plaques. The regulation of GRIA2 in vascular smooth muscle cells (VSMCs) was confirmed by lentiviral transfection. Overexpression of GRIA2 promoted the proliferation and migration of VSMCs. Using Kyoto Encyclopedia of Genes and Genomes (KEGG) enrichment analysis and protein-protein interaction (PPI) network, a strong connection between ENPP3 and GRIA2 was discovered. In vitro results showed that the high expression of GRIA2 in VSMCs enhanced the expression of ENPP3, while downregulation of GRIA2 downregulated ENPP3. GRIA2 is highly differentially expressed in restenotic arterial plaques, promoting the proliferation and migration of VSMCs through upregulation of ENPP3. These discoveries will help us to obtain a better understanding of restenosis in lower extremity arteries.

Keywords: restenosis, GRIA2, percutaneous transluminal angioplasty, peripheral artery disease, vascular smooth muscle cells

\section{INTRODUCTION}

Peripheral artery disease (PAD) affects approximately $15-20 \%$ of persons over 70 years of age (Criqui et al., 1985; Hiatt et al., 1995; Selvin and Erlinger, 2004), and chronic atherosclerotic ischemia of the lower extremities leads to a high amputation rate and mortality (Diehm et al., 2009; Zeller et al., 2009; Meves et al., 2010). Despite the wide use of percutaneous transluminal angioplasty (PTA), due to its minimal invasiveness and effectiveness (Apelqvist et al., 2011), restenosis occurs in up to $70 \%$ of patients within 1 year (Liistro et al., 2013) and limits the 
long-term efficacy. Although there have been attempts to prevent or reduce restenosis pharmacologically through drug-coated balloons (DCBs; Schmidt et al., 2016; Peterson et al., 2017; Liistro et al., 2019), the restenosis rate is still high with almost $23 \%$ of patients developing it within 12 months (Liistro et al., 2019). Restenosis has become the main obstacle to long-term efficacy post-PTA in lower extremity arteries. Elucidating the regulatory mechanism of restenosis post-PTA will aid in significantly improving PTA prognosis and the long-term patency rate of lower extremity arteries.

Excessive proliferation and migration of vascular smooth muscle cells (VSMCs) are the predominant pathogenic mechanisms of the restenosis process (Bennett et al., 2016). Usually, mature VSMCs maintain a contractile-like resting state and stay in the media of the vessels. PTA damages the vascular intima and causes physical injury thereby inducing the conversion of VSMCs to a proliferative phenotype followed by migration to the intima and proliferation, leading to restenosis (Newby and Zaltsman, 2000; Louis and Zahradka, 2010; Gomez and Owens, 2012). Despite decades of study, a sufficient understanding of restenosis pathogenesis remains elusive, which is why developing targeted therapeutic strategies has been difficult. Clarifying the mechanism of VSMCs migration and proliferation in restenosis may provide new research insights into the causes of restenosis, which may lead to treatment strategies that ameliorate the overall efficacy of PTA treatment.

Recent advances have facilitated efforts to determine restenosis pathogenesis. Gene sequencing technology can verify molecular abnormalities correlated with pathophysiology and identify therapeutic targets for diverse diseases (Grunewald et al., 2015; LeBlanc and Marra, 2015). Searching the Gene Expression Omnibus (GEO) database for data related to the development and progression of restenosis has helped researchers to track restenosis pathogenesis (Rabbani et al., 2016). In our study, two datasets (GSE23314 and GSE53274) were chosen from the GEO database. The two datasets included 11 restenotic (RS) plaque tissues and 99 atherosclerotic (AS) plaque tissues. The gene expression profiling data from these tissues were reanalyzed, and differentially expressed genes (DEGs) were identified. In addition to analyzing Gene Ontology (GO) enrichment data, glutamate ionotropic receptor AMPA type subunit 2 (GRIA2), which is a glutamate receptor (Hansen et al., 2007), was found to be significantly correlated with restenosis pathogenesis. Moreover, using Kyoto Encyclopedia of Genes and Genomes (KEGG) enrichment analysis, ENPP3 was predicted to be the downstream target of GRIA2, participating in the proliferation and migration of VSMCs in restenosis.

\footnotetext{
Abbreviations: PTA, Percutaneous transluminal angioplasty; PAD, Peripheral artery disease; DEGs, Differentially expressed genes; GEO, Gene Expression Omnibus; GO, Gene Ontology; KEGG, Kyoto Encyclopedia of Genes and Genomes; VSMCs, Vascular smooth muscle cells; HVSMCs, Human vascular smooth muscle cells; DCBs, Drug-coated balloons; GRIA2, Glutamate ionotropic receptor AMPA type subunit 2; SD, Sprague-Dawley; qRT-PCR, Quantitative real-time polymerase chain reaction; EdU, 5-Ethynyl-2'-deoxeuridine; AS, Atherosclerotic; RS, Restenotic; PPI, Protein-protein interaction; ENPP3, Ectonucleotide pyrophosphatase/ phosphodiesterase 3; PVDF, Polyvinylidene fluoride.
}

\section{MATERIALS AND METHODS}

\section{Data Collection and Microarray Data}

We searched the GEO database for publicly available studies using the following keywords: "atherosclerosis OR restenosis OR restenotic plaque OR atherosclerotic plaque" (study keyword), "Homo sapiens" (organism), and "Expression profiling by array" (study type). Databases were searched for all data reported before July 30, 2020. The inclusion criteria were as follows: (1) restenosis tissue samples and arteriosclerotic lower extremity artery tissue samples and (2) mRNA expression profiles. After systematic analysis, two mRNA gene expression profiles (GSE23314 and GSE53274) were reviewed further. The GSE23314 dataset included six restenotic tissue samples and 95 arteriosclerotic tissue samples. The GSE53274 dataset contained four arteriosclerotic tissue samples and five restenotic tissue samples. The detection platforms used for mRNA sequencing of GSE23314 and GSE53274 were GPL4372 and GPL570 (Affymetrix), respectively.

\section{DEGs Data Processing}

To identify common DEGs in the datasets, we used GEO2R, which is an interactive web tool that compares two or more groups of samples under the same experimental conditions in a GEO dataset. ${ }^{1}$ All the DEGs were identified according to the inclusion criteria of $p<0.05$. Bioinformatics \& Evolutionary Genomics, which is an online website tool, was used to plot Venn diagrams of these two datasets. Each circle represents a dataset, and the overlapping area represents the target DEGs.

\section{GO and KEGG Pathway Enrichment Analyses of DEGs}

The functional and pathway enrichment of the proteins encoded by candidate genes were analyzed, and these genes were annotated using the DAVID database. ${ }^{2} \mathrm{GO}$ enrichment analysis of the molecular functions of the DEGs was performed using the DAVID database. KEGG analysis of the DEGs was performed using the KOBAS online analysis database. ${ }^{3}$

\section{Protein-Protein Interaction Network Integration}

Cytoscape is a software system that is commonly used to identify the interactions between known proteins and predicted proteins (Doncheva et al., 2019). Each node is a gene or protein, and the connections between nodes represent the interactions between these biological molecules, which can be used to identify interactions and pathway relationships between the proteins encoded by the DEGs in our research. The corresponding proteins in the central node may be core proteins or key candidate genes with important physiological regulatory functions.

\footnotetext{
www.ncbi.nlm.nih.gov/geo/geo2r

${ }^{2} \mathrm{https} / /$ david.ncifcrf.gov/

${ }^{3}$ http://kobas.cbi.pku.edu.cn/
} 


\section{Animal Model}

Male Sprague-Dawley (SD) rats ( 120g) were obtained from the Beijing Huafukang Bioscience Co., Inc. in China. During the entire study, all rats had free access to water and food. The animal care and experiments were performed in accordance with those in previous publications (Zhou et al., 2015, 2016). The protocol was authorized by the Ethics Committee of Xuanwu Hospital affiliated with Capital Medical University. All rats underwent surgical procedures under anesthesia with sodium pentobarbital.

A high-fat diet was provided to all rats throughout the experiment. All eligible rats were assigned to two groups: restenosis and atherosclerosis groups. To emphasize restenotic plaque alternations, rats from the atherosclerosis group were chosen as the control. The surgical procedures for the establishment of restenosis $(n=6)$ and atherosclerosis $(n=6$; control group) models were as follows: For the restenosis group, first, a $1.5-\mathrm{mm}$ wire-guided balloon catheter (Medtronic, Inc., Minnesota, America) was inserted into the iliac artery through the femoral artery. Then, endothelial denudation was achieved by inflating balloon catheters at 8,10 , and 12 atmospheric pressure (atm). Approximately 4 weeks after the first surgical procedure, PTA was further performed for the induction of restenotic plaques if the iliac artery developed severe atherosclerotic damage, which was confirmed by ultrasound examination. Then, a color Doppler ultrasonogram was used to examine the formation of the stenosed iliac arteries after PTA. For the atherosclerosis group, rats received a sham surgery on the same day as the first surgical injury in the restenosis group, and balloon-induced injuries of the iliac arteries were performed to develop models of atherosclerosis after 4 weeks (Zhou et al., 2015, 2016; Zou et al., 2020).

\section{Double Immunofluorescence Staining}

Paraffin-embedded tissue sections were cut into slices $(4 \mu \mathrm{m})$, were deparaffinized in xylene, and were rehydrated in graded alcohol solutions. The slides were blocked antigen with 5\% $(\mathrm{v} / \mathrm{v})$ goat serum for $1 \mathrm{~h}$. After $1 \times$ PBS wash, specimens were then stained with rabbit anti-GRIA2 (1:100; Abcam, Cambridge, United Kingdom) and rabbit anti- $\alpha-S M A$ (1:200, Abcam, Cambridge, United Kingdom) antibodies at $4^{\circ} \mathrm{C}$ overnight. After rinsing with PBS for three times, immunoreactivity products were visualized by incubation with appropriate Alexa Fluor 488-conjugated secondary antibodies (1:200; Thermo Fisher Scientific, Waltham, MA, United States), 594-conjugated secondary antibodies (1:200; Thermo Fisher Scientific, Waltham, MA, United States), and DAPI $(1 \mu \mathrm{g} / \mathrm{ml}$, Solarbio, Beijing, China) to visualize cell nuclei. After rinsing, specimens were examined with a fluorescence microscope (OLYMPUS FSX100). ImageJ software (National Institutes of Health, https://imagej. net/Citing) was used to quantify the fluorescence intensity of the cells in the intima.

\section{Immunohistochemical Analysis}

Tissue sections deparaffinized in xylene and rehydrated with graded alcohol solutions were incubated with $1 \% \mathrm{H}_{2} \mathrm{O}_{2}$ in methanol for 10 min to block endogenous peroxidase activity. The slides were blocked antigen with $5 \%(\mathrm{v} / \mathrm{v})$ goat serum for $1 \mathrm{~h}$, and incubated with the primary antibodies (rabbit anti-ENPP3, 1:100, Affinity Biosciences, United States) overnight at $4^{\circ} \mathrm{C}$. After rinsing with $\mathrm{PBS}$, the sections were incubated with peroxidase enzyme-conjugated goat anti-rabbit secondary antibody (\#PV9001, Zhongshan, Beijing, China) for $30 \mathrm{~min}$ at $37^{\circ} \mathrm{C}$. Diaminobenzidine tetrahydrochloride (ZSBIO, Beijing, China) in PBS was used to produce a brown color. Then, the sections were counterstained with haematoxylin. ImageJ software (National Institutes of Health, https://imagej.net/ Citing) was used to quantify the optical density (OD) values in the intima.

\section{Cell Culture and Transfection}

Human vascular smooth muscle cells (HVSMCs) were purchased from Shanghai Zhong Qiao Xin Zhou Biotechnology Co., Ltd. The GRIA2 lentiviral vector was purchased from GeneChem Corporation (Shanghai, China). HVSMCs at $30-40 \%$ confluency were transfected with Lenti-GRIA2-shRNA, Lenti-GRIA2 or Lenti-NC-shRNA, according to the manufacturer's protocol (GeneChem, Shanghai, China). After $48 \mathrm{~h}$ of transfection, the cells were treated with $1 \mu \mathrm{g} / \mathrm{ml}$ puromycin for 1 week for the selection of stably expressing cells. The HVSMCs with stable knockdown of GRIA2 were established. RNA was extracted and real-time (RT)-PCR experiments were conducted to confirm the efficiency of the intervention.

HVSMCs with Lenti-GRIA2 were transiently transfected with the ENPP3 plasmids (GeneChem, Shanghai, China) or the empty control plasmids. Briefly, Opti-MEM (Gibco-BRL/Invitrogen, Carlsbad, CA), containing $2.5 \mu \mathrm{g}$ of the ENPP3 plasmids or the empty plasmids was mixed with $5 \mu$ l of Lipofectamine 3000 transfection reagent (Thermo Fisher Scientific, Waltham, MA, United States), and the mixture was added to the cells. After 8-12 h, the Opti-MEM was removed. Forty-eight hours after transfection, the transfection efficiency was evaluated by RT-PCR and western blotting.

Following the manufacturer's instructions, the siRNAs against ENPP3 were transfected into HVSMCs using Lipofectamine 2000 transfection reagent (Thermo Fisher Scientific, Waltham, MA, United States) after transfection with Lenti-GRIA2-shRNA. The siRNA knockdown efficiency was verified by RT-PCR and western blotting.

\section{Quantitative Real-Time Polymerase Chain Reaction}

Total RNA was extracted from HVSMCs using TRIzol total RNA extraction reagent (Invitrogen, United States). cDNA was synthesized using a Light Cycler kit (Takara, Japan). $G A P D H$ was used as an endogenous control for each sample. The relative expression level of the target genes was analyzed

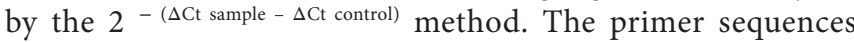
were as follows: GRIA2 F: AAAGAATACCCTGGAGCA CAC and R: CCAAACAATCTCCTGCATTTCC; ENPP3 
F: CGACTGCACTATGCCAAGAA and R: CATGGGCATCCTC ATAGCTT; and GAPDH F: AGGTCGGTGTGAACGGATTTG and R: GGGGTCGTTGATGGCAACA.

\section{Proliferation and Migration Assays}

The effect of GRIA2 on cell proliferation was tested by the 5-ethynyl-2' -deoxeuridine (EdU) assay kit (RiboBio Co., Ltd., Wuhan, China). First, HVSMCs were resuspended at $5 \times 10^{3}$ cells $/ 100 \mu \mathrm{l}$ and seeded into 96-well plates (Costar, Solarbio, Beijing, China) after transfection with Lenti-GRIA2, LentiGRIA2-shRNA, and Lenti-NC-shRNA. Then, the cells were incubated with EdU $(50 \mu \mathrm{m})$ at $37^{\circ} \mathrm{C}$ for $2 \mathrm{~h}$. Then, the VSMCs were fixed with $4 \%$ formaldehyde for $30 \mathrm{~min}$, incubated with glycine $(2 \mathrm{mg} / \mathrm{ml})$ for $5 \mathrm{~min}$, and treated with $0.5 \%$ Triton X-100 for $10 \mathrm{~min}$. After a PBS wash, cells were incubated with $100 \mu$ l of $1 \times$ Apollo reaction cocktail for $30 \mathrm{~min}$ and treated with $0.5 \%$ Triton X-100. DNA was stained with Hoechst 33342 stain for $30 \mathrm{~min}$ to stain all the nuclei and was then visualized with an Olympus FSX100 imaging system (Olympus, Tokyo, Japan). Five groups of confluent cells were randomly selected from each sample image. The assay was repeated at least three times.

The regulation of cell migration by GRIA2 was verified using a 24-well Transwell (Corning-Costar, United States), and membrane insets were coated with cells. After transfection with Lenti-GRIA2, Lenti-GRIA2-shRNA, and Lenti-NCshRNA, HVSMCs at $2 \times 10^{4}$ cells $/ 100 \mu \mathrm{l}$ were seeded in the upper chambers of Matrigel-coated 8- $\mu \mathrm{m}$ pore size Transwell filters (Corning Life Sciences, United States) while 10\% serum culture medium was placed in the bottom of the plates. After incubation for $24 \mathrm{~h}$, the cells that migrated to the lower surface of the filter were fixed in $4 \%$ paraformaldehyde for $15 \mathrm{~min}$ and stained with crystal violet, and the nonmigrating cells in the upper surface of the filter were removed with a cotton-tipped swab. Images of migrated cells were taken at randomly selected fields using an Olympus FSX100 imaging system (Olympus, Tokyo, Japan). Five groups of confluent cells were randomly selected from each sample image and analyzed. The assay was repeated at least three times.

\section{Western Blot Analysis}

Protein concentrations were determined using a Bio-Rad DC Protein Assay kit (Bio-Rad Laboratories, Inc., Hercules, CA, United States). Equal amounts of protein were resolved on $10 \%$ SDS polyacrylamide gels and transferred to polyvinylidene fluoride (PVDF) membranes (EMD Millipore, Billerica, MA, United States). The membranes were subsequently incubated with rabbit anti-GRIA2 (1:100; Abcam, Cambridge, United Kingdom), rabbit anti-ENPP3 (1:1,000, ab190823, Abcam, Cambridge, United States), or mouse anti- $\beta$-actin (1:1000; A1978, Sigma Aldrich; Merck KGaA) overnight at $4{ }^{\circ} \mathrm{C}$. The next day, the membranes were incubated with the corresponding peroxidase-labeled goat anti-rabbit (1:10,000, Abcam, Cambridge, United States) or anti-mouse secondary antibodies (1:10,000, Abcam, Cambridge,
United States). ImageJ software (National Institutes of Health, https://imagej.net/Citing) was used to quantify the densitometry of the bands.

\section{Statistical Analysis}

All data were analyzed using GraphPad Prism 5 software (GraphPad Software, Inc., San Diego, CA). Student's $t$-test was used to assess significance within two groups. One-way or two-way ANOVA analysis was used, where appropriate to assess the differences for multiple comparisons. All data are presented as the means \pm SEM, and the statistical significance was considered as $p<0.05$.

\section{RESULTS}

\section{Data Mining: Integrated Analysis of Two GEO Datasets Identified Significant DEGs in Atherosclerotic and Restenotic Arterial Plaques}

In this study, we performed multistep analysis to explore key DEGs and their significant biological functions in restenosis using integrated bioinformatics methods. First, we identified two GEO datasets (GSE23314 and GSE53274) that included RS tissue samples and AS tissue samples (11 RS samples and 99 AS samples). On the basis of the cut-off criteria, DEGs in each GEO dataset were identified between RS and AS. There were 4,146 DEGs in GSE23314, including 2,667 upregulated and 1,479 downregulated genes, and 12,960 DEGs in GSE53274, including 2,758 upregulated and 10,202 downregulated genes. Among these, we only found 1852 common DEGs between GSE23314 and GSE53274. The intersection plot of DEGs in these datasets is shown in Figure 1A. To characterize the functional roles of the identified DEGs, we used GO and KEGG pathway enrichment analysis. The GO analysis results showed that the DEGs were significantly enriched in the ionotropic glutamate receptor signaling pathway and synaptic transmission and glutamatergic pathways.

KEGG pathway analysis was performed using the KOBAS 3.0 system, and the DEGs were enriched in metabolic pathways. Analysis of 153 common DEGs in metabolic pathways was performed using Cytoscape, suggesting that 137 proteins interacted, among which the first 10 proteins with more connections in nodes were "ENPP3," "CYP2E1," "YP2A4," "ENTPD3," "ADSL," "PDE3B," "ADCY," "CANT," "ADH6," "ADH4" and "MGST1." ENPP3 (black arrow) was the most important protein, connecting a total of 23 proteins, as shown in Figure 1B.

\section{GRIA2 Is Highly Expressed in VSMCs in Restenotic Arteries}

Through the analysis of mRNA expression in the GEO database, we found that GRIA2 was highly expressed in restenosis tissues. Combined with the analysis of the plaque tissues, GRIA2 expression in restenotic plaques was significantly higher than that in atherosclerotic plaques. In addition, double immunofluorescence staining of GRIA2 


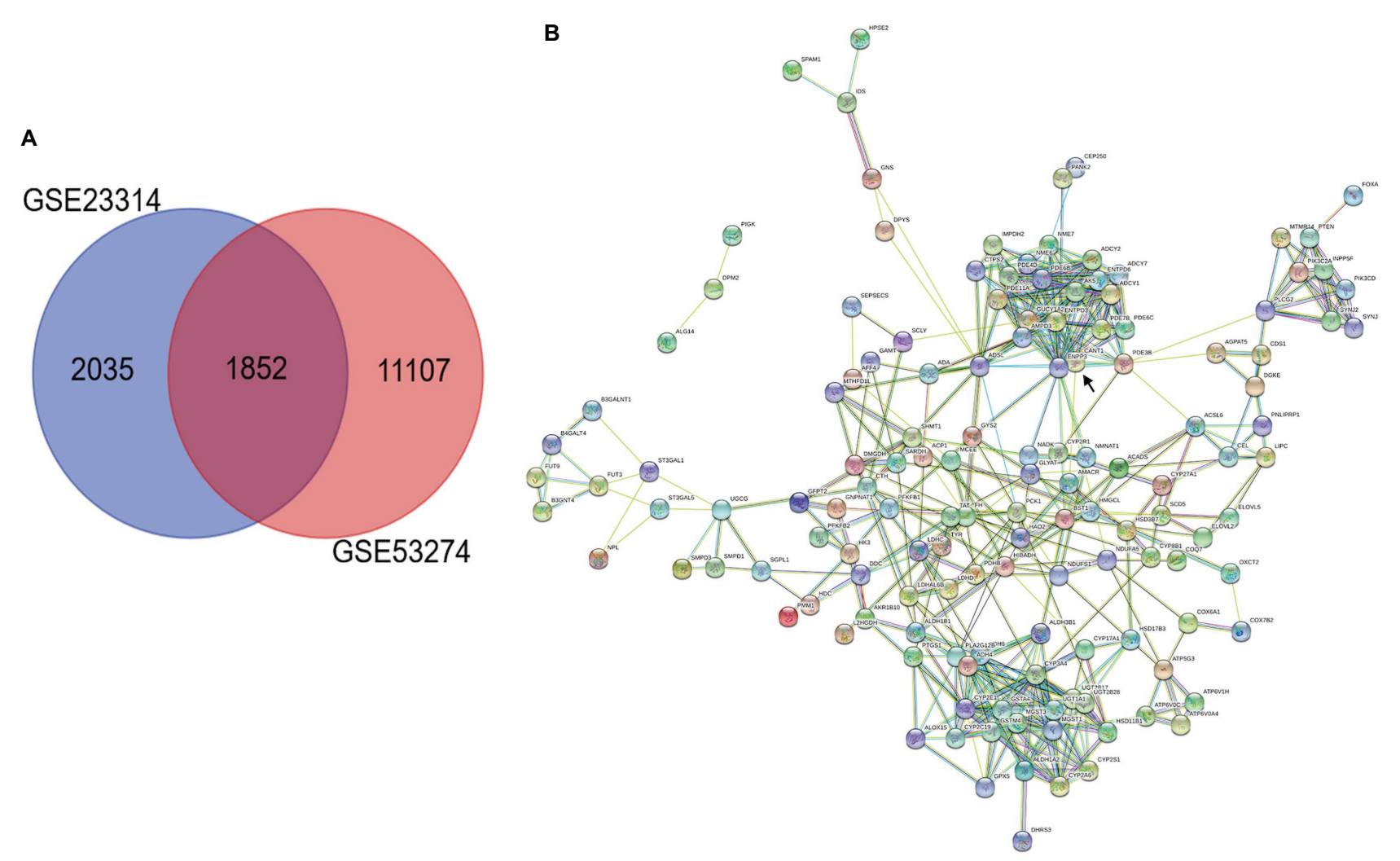

FIGURE 1 | Integrated analysis of GEO datasets identified DEGs. (A) Venn diagrams of common DGEs between two combined datasets (GSE23314 and GSE53274). Each circle represents a dataset, and the overlap between the circles is the overlap between the datasets. (B) PPI network integration of the common DGEs. Each node is a gene, and the connections between nodes represent the interaction of these biological molecules. ENPP3 (black arrow).

and $\alpha-S M A$ in restenosis plaque tissues revealed that the red GRIA2 fluorescence staining in the intima was stronger than that of atherosclerotic tissues $(69.2 \pm 5.3,11.5 \pm 3.6$ for RS, AS, $p<0.01)$. The green $\alpha$-SMA fluorescence staining $(75.5 \pm 8.1,22.8 \pm 5.6$ for RS, AS, $p<0.05)$, which is a marker of VSMCs, colocalized with the red fluorescence staining in restenotic tissues, indicating that GRIA2 exerts its function through VSMCs. Therefore, we concluded that GRIA2 might acts as an activator in restenosis and is associated with VSMCs behavior (Figure 2).

\section{GRIA2 Is Associated With the Proliferation and Migration of VSMCs in vitro}

The mRNA $(0.43 \pm 0.17,4.37 \pm 0.51$ for Lenti-GRIA2-shRNA, Lenti-GRIA2 vs. $1.09 \pm 0.13$ for NC, $p<0.01)$ and protein expression levels $(0.65 \pm 0.09,3.16 \pm 0.23$ for Lenti-GRIA2shRNA, Lenti-GRIA2 vs. $1.21 \pm 0.15$ for NC, $p<0.01$ ) of GRIA2 significantly decreased in VSMCs transfected with LentiGRIA2-shRNA, and were upregulated in Lenti-GRIA2 transfected VSMCs (Figures 3A,B). EdU assays were used to assess the proliferation ability of VSMCs. Figures 3C,D shows that VSMCs overexpressing GRIA2 grew faster than the Lenti-NC-shRNA group $(71.5 \pm 5.09$ for Lenti-GRIA2 vs. $50.7 \pm 3.85$ for NC, $p<0.01$ ), and cells with GRIA2 knockdown exhibited a slower rate of proliferation $(36.8 \pm 3.83$ for LentiGRIA2-shRNA vs. $50.7 \pm 3.85$ for NC, $p<0.01$ ). Additionally, cell migration Transwell assays were conducted. Upregulation of GRIA2 led to a marked increase in VSMCs migration $(79.2 \pm 3.58$ for Lenti-GRIA2 vs. $41.3 \pm 3.82$ for NC, $p<0.001)$, and downregulation of GRIA2 led to a significant decrease $(22.1 \pm 2.85$ for Lenti-GRIA2-shRNA vs. $41.3 \pm 3.82$ for $\mathrm{NC}$, $p<0.01$; Figures 3E,F).

\section{GRIA2 Interacted With ENPP3 in Restenosis}

To gain more insight into the role of GRIA2 in restenosis, we conducted in-depth mining. KEGG enrichment analysis showed that the 1852 common DEGs in restenosis and atherosclerosis tissue samples were significantly enriched in metabolic pathways. Then, a protein-protein interaction (PPI) network was constructed. Network analysis of protein interaction data showed that ectonucleotide pyrophosphatase/ phosphodiesterase 3 (ENPP3) acts as the central node. By reviewing the literature, we then predicted ENPP3 as the downstream target of GRIA2. During in vivo experiments, immunohistochemical analysis results showed that ENPP3 was significantly expressed extremely higher in restenotic plaques $(10.32 \pm 1.25,3.51 \pm 0.55$ for RS, AS, $p<0.05$; Figure 4A). 
A

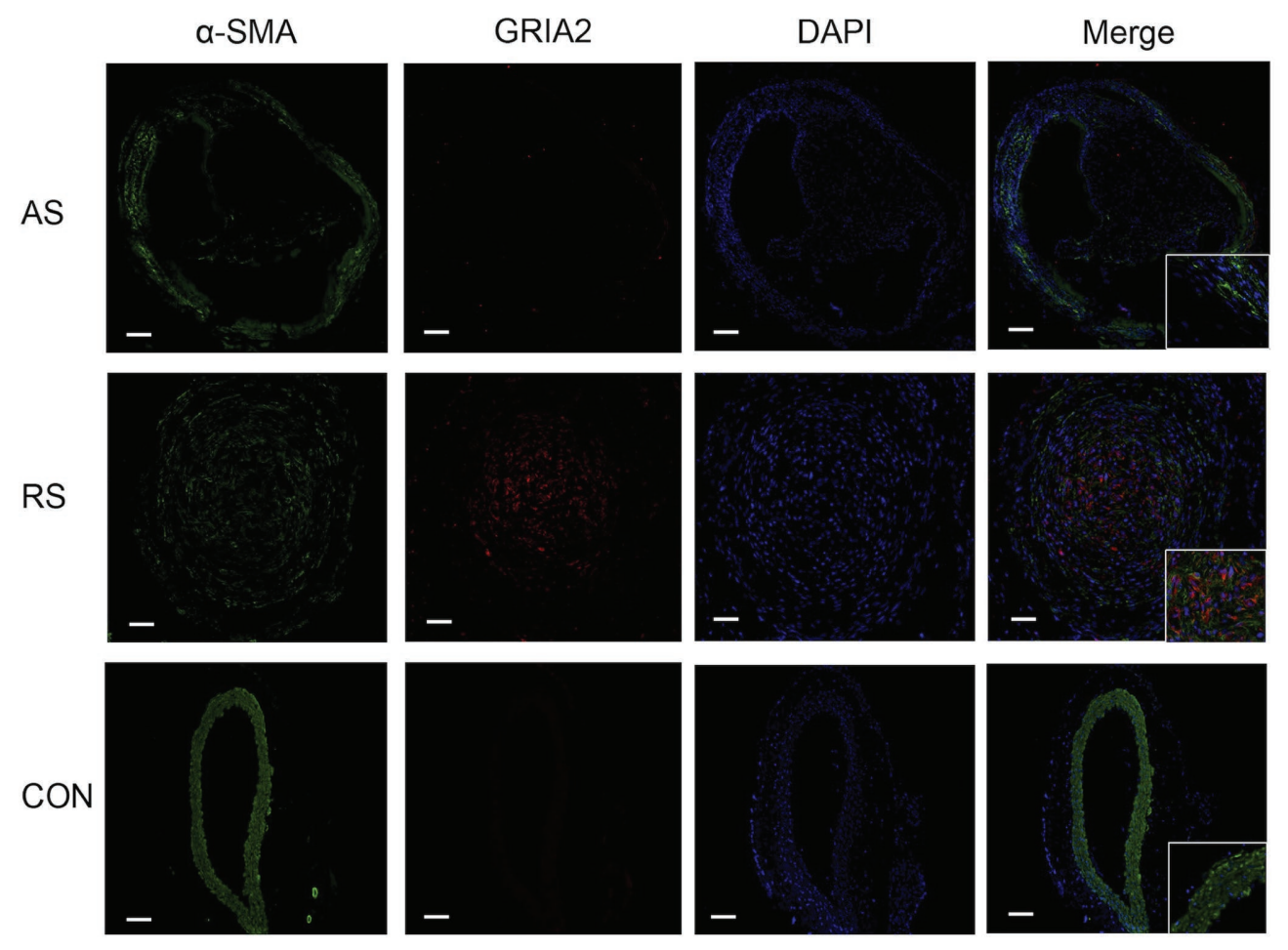

B
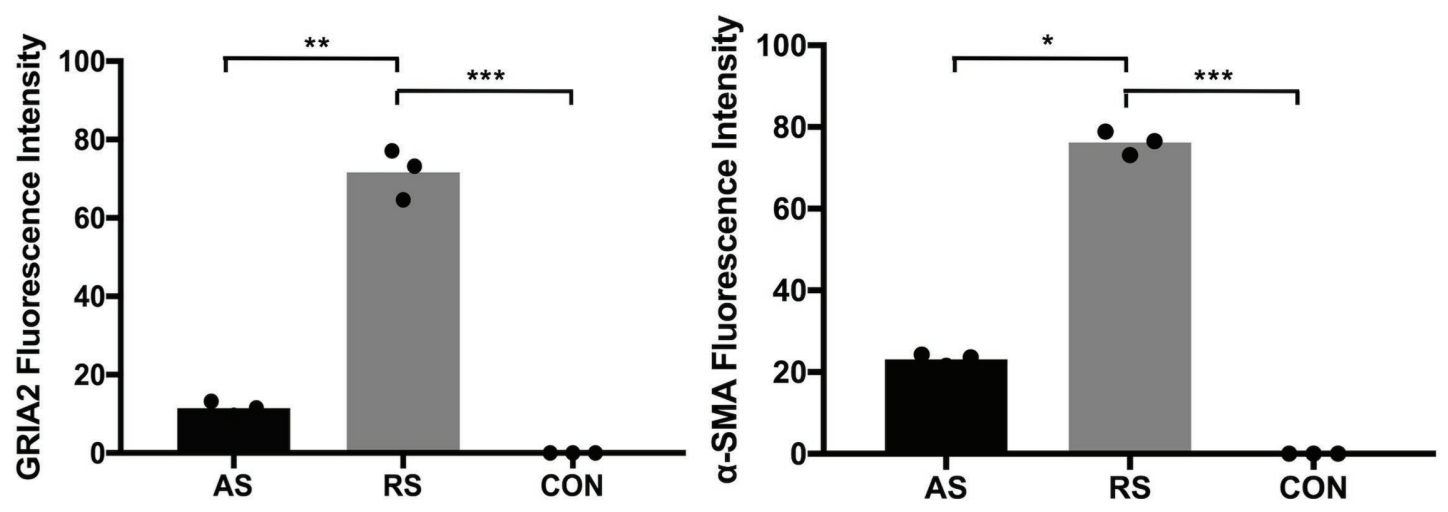

FIGURE 2 | GRIA2 is highly expressed in VSMCs in restenosis. (A) Double immunofluorescence staining of GRIA2 (red) and VSMCs (green, stained with $\alpha$-SMA) in restenotic plaque tissues, atherosclerotic plaque tissues and healthy control artery tissues is shown $(n=3)$. Intensity of fluorescence is the statistical calculations in the intima. Images are representative, and (B) the bar graph shows the average data of three independent experiments $(n=3)$. Staining was performed at $100 \times$ magnification; the right part of the picture was magnified to $200 \times$ (scale bar, $50 \mu \mathrm{m})$. AS: atherosclerotic; RS: restenotic; CON: control; VSMCs: vascular smooth muscle cells. ${ }^{*}$ Statistically significant difference $(p<0.05)$; ${ }^{* \star}$ Statistically significant difference $(p<0.01)$; ${ }^{* \star}$ Statistically significant difference $(p<0.001)$.

The analysis of mRNA expression showed that ENPP3 was significantly higher in restenotic plaques than in atherosclerotic plaques (3.31 \pm 0.37 for RS, vs. $1.13 \pm 0.25$ for AS, $p<0.001$; Figure 4A). In cell lines, the high expression of GRIA2 in VSMCs enhanced the expression of ENPP3 $(2.29 \pm 0.36$ for Lenti-GRIA2 vs. $1.47 \pm 0.16$ for Control, $p<0.05)$, while downregulation of GRIA2 downregulated ENPP3 $(0.37 \pm 0.11$ for Lenti-GRIA2-shRNA vs. $1.47 \pm 0.16$ for Control, $p<0.01$ ), which was consistent with the results in vivo (Figure 4B).

\section{The Promotion of VSMCs Proliferation and Migration by GRIA2 Is Reversed by ENPP3 in vitro}

Based on the regulation of ENPP3 by GRIA2, we further investigated the effects of GRIA2-ENPP3 on VSMCs biological functions. The results of EdU and Transwell assays showed that compared with the control group, the overexpression of GRIA2 significantly promoted cell proliferation and migration; however, with concomitant downregulation of ENPP3, the promoted cell 
A

C

GRIA2

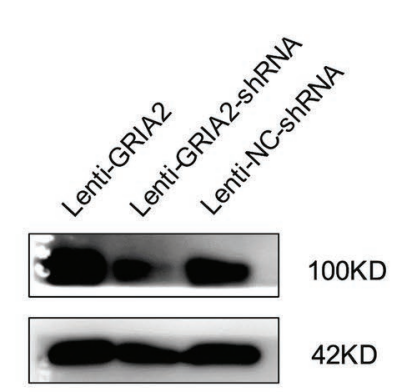

$\beta$-actin

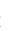

Lenti-GRIA2-shRNA

$$
\text { Lenti-GRIA2 }
$$

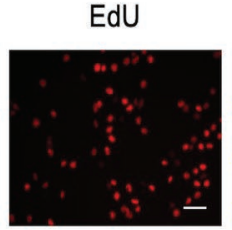

Lenti-NC-shRNA

E
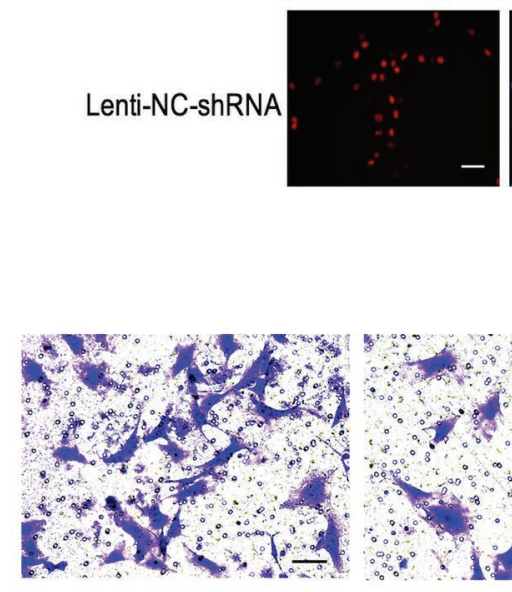

Lenti-GRIA2

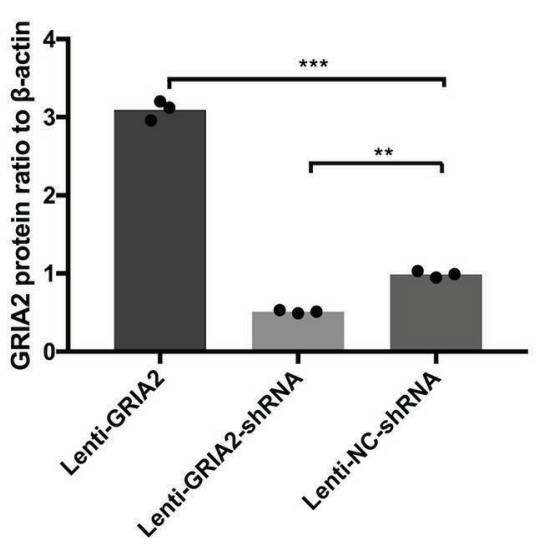

Hoechst $33342 \quad$ Merge
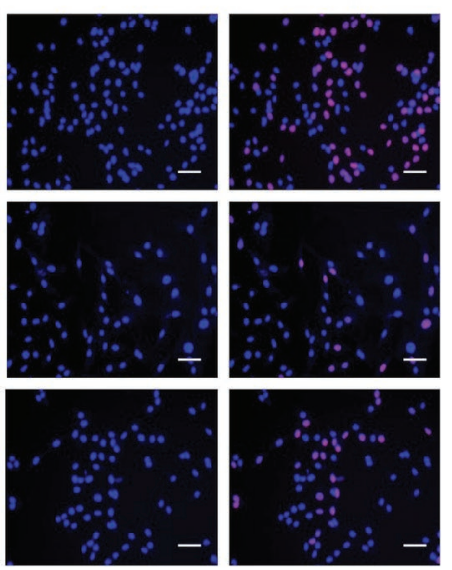

B

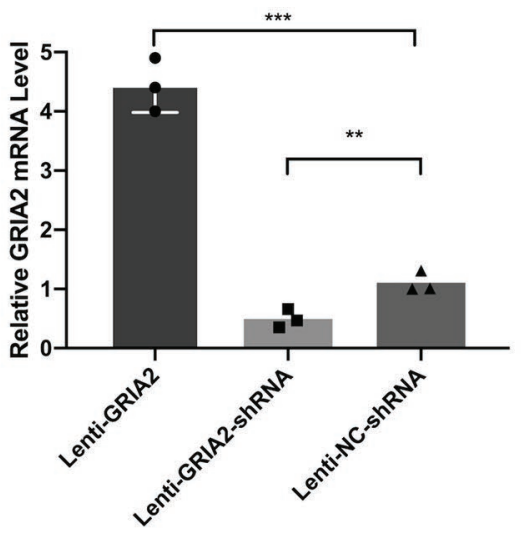

D

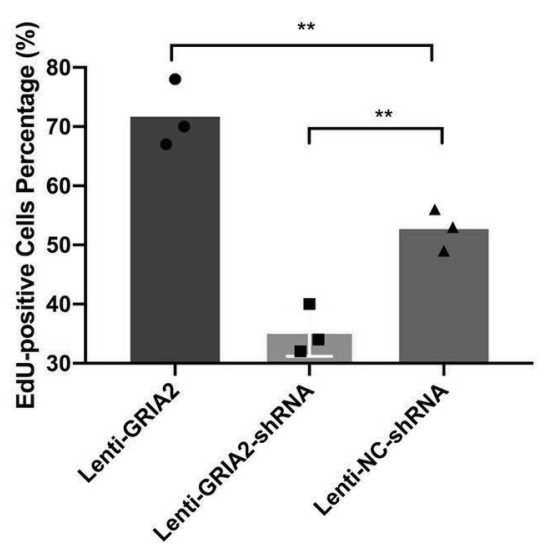

F

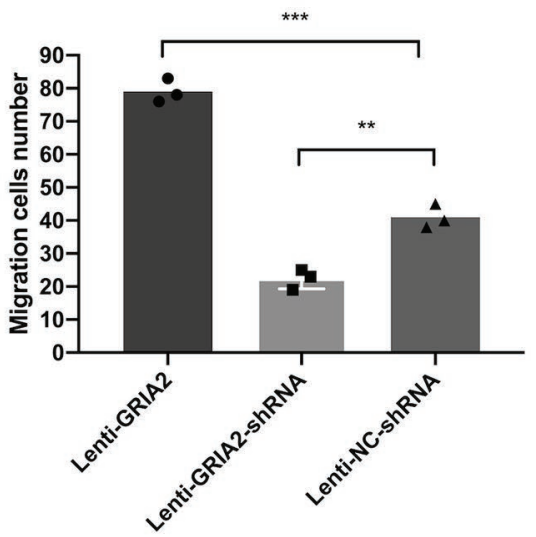

FIGURE 3 | The regulation of GRIA2 in VSMCs. (A) GRIA2 protein expression was detected by western blot after cDNA and shRNA regulation of GRIA2. Blots are representative, and the bar graph shows the average data $(n=3)$. (B) GRIA2 mRNA expression was detected by real-time PCR after transfection with lentivirus carrying GRIA2 shRNA, cDNA or empty vector $(n=3)$. (C) EdU assay showed that increased EdU-positive VSMCs overexpressing GRIA2 were observed (scale bar, $50 \mu \mathrm{m})$. Images are representative, and the bar graph shows the average data of independent experiments $(n=3)$. (D) Rate of EdU-positive VSMCs ( $n=3)$.

(E) Transwell migration assay of VSMCs after upregulating GRIA2 expression (scale bar, $100 \mu \mathrm{m})$. Images are representative, and the bar graph shows the average data of three independent experiments $(n=3)$. (F) Quantitative data on the migration of VSMCs $(n=3)$. ${ }^{* *}$ Statistically significant difference $(p<0.01)$ and ${ }^{* * *}$ statistically significant difference $(p<0.001)$

proliferation $(38.9 \pm 3.88$ for Lenti-GRIA2 + siENPP3 vs. $76.2 \pm 5.91$ for Lenti-GRIA2 + siNC, $p<0.01)$ and migration $(33.2 \pm 2.69$ for Lenti-GRIA2+siENPP3 vs. $73.3 \pm 4.01$ for Lenti-GRIA2+siNC, $p<0.001)$ abilities were reversed. Downregulation of GRIA2 expression decreased VSMCs proliferation $(29.2 \pm 2.63$ for LentiGRIA2-shRNA + NC plasmids vs. $68.1 \pm 4.35$ for Lenti-GRIA2shRNA + ENPP3 plasmids, $p<0.001)$, and migration $(22.5 \pm 2.39$ for Lenti-GRIA2-shRNA+NC plasmids vs. $73.7 \pm 5.34$ for 


\section{A}
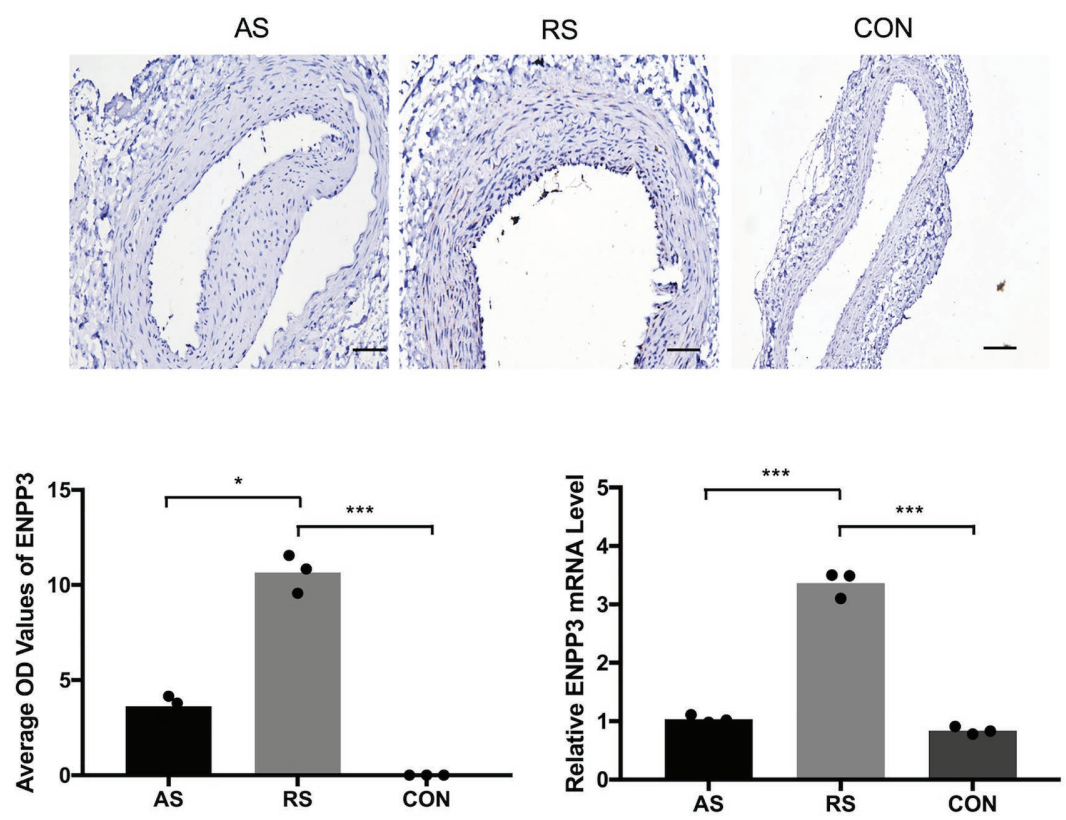

B

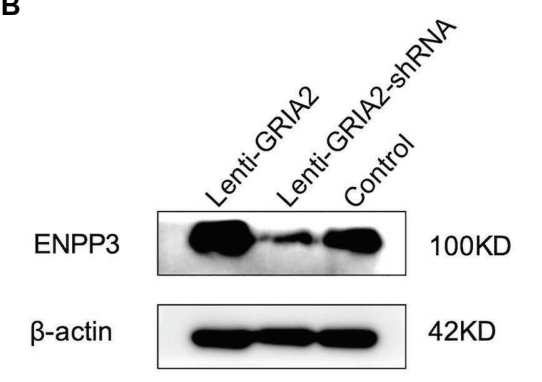

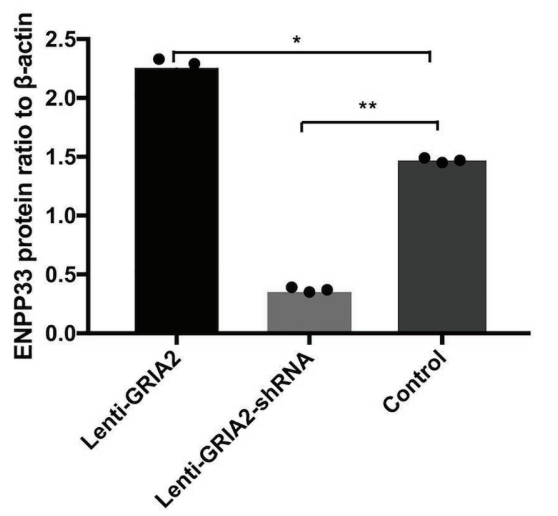

FIGURE 4 | GRIA2 interacted with ENPP3 in restenosis. (A) Immunohistochemical analysis and quantitative mRNA analysis showed the expression of ENPP3 in restenotic plaque tissues, atherosclerotic plaque tissues and healthy control artery tissues $(n=3)$. Optical density (OD) values are the statistical calculations in the intima. Images are representative, and the bar graph shows the average data of three independent experiments $(n=3)$. Staining was performed at $200 \times$ magnification (scale bar, $50 \mu \mathrm{m}$ ). (B) The expression of ENPP3 in VSMCs after upregulating GRIA2 expression. Blots are representative, and the bar graph shows the average data $(n=3)$. ${ }^{*}$ Statistically significant difference $(p<0.05)$, ${ }^{* *}$ statistically significant difference $(p<0.01)$, and ${ }^{* *}$ statistically significant difference $(p<0.001)$. AS: atherosclerotic; RS: restenotic; CON: control; VSMCs: vascular smooth muscle cells.

Lenti-GRIA2-shRNA+ENPP3 plasmids, $p<0.001)$ was increased by ENPP3 overexpression (Figures $\mathbf{5 A}-\mathbf{D}$ ). In summary, our experiments showed that ENPP3 inhibits GRIA2-induced VSMCs.

\section{DISCUSSION}

Restenosis of lower extremity arteries after PTA is an unsolved conundrum. The goal of the current study was to identify candidate driver genes in restenosis through the analysis of multiple datasets of gene expression arrays. By screening, GSE23314 and GSE53274 were chosen, and through the analysis of the two datasets, we discovered significant differences in GRIA2 expression in restenosis and atherosclerosis tissues. Moreover, GRIA2 levels in restenosis were confirmed to be highly expressed in VSMCs in the intima. Meanwhile, in VSMCs, GRIA2 was demonstrated to be a promoting factor of the proliferation and migration. Furthermore, we also verified the downstream target of GRIA2. By KEGG enrichment analysis, ENPP3 was predicted. A series of experimental results revealed that GRIA2 regulates ENPP3 and modulates VSMCs proliferation and migration through ENPP3.

GRIA2, which encodes an AMPA-selective ionotropic glutamate receptor subunit (also known as GLUR2), affects cell membrane calcium permeability (Lee et al., 2010; Liu et al.,
2010; Oakes et al., 2017; Tischbein et al., 2019; Konen et al., 2020), cell proliferation, motility, and cell death (Yoshioka et al., 1996; Takeda et al., 2000; Rzeski et al., 2001; Abdul and Hoosein, 2005; Zhang et al., 2017). GRIA2 is normally expressed in the central nervous system under physiological conditions (Song et al., 1998; Liu and Cull-Candy, 2000; Nakagawa et al., 2005), but GRIA2 can be detected in various oncogenic conditions through unknown mechanisms. Abnormal GRIA2 expression has also been demonstrated in several tumors and tumor cell lines (Yoshioka et al., 1996; Takeda et al., 2000; Rzeski et al., 2001; Abdul and Hoosein, 2005; Ishiuchi et al., 2007; Ni et al., 2020) and is thought to mediate increased cell proliferation.

GRIA2 expression has not yet been characterized in restenosis. We discovered, for the first time, that GRIA2 accumulated in restenotic plaques and may participate in the restenosis process. VSMCs were the dominant components of restenotic plaques. The migration and proliferation of VSMCs are critical events in the development of restenosis (Bennett et al., 2016). We verified the function of GRIA2 in VSMCs. The results showed that overexpression of GRIA2 significantly promoted the proliferation and migration of VSMCs, and downregulation of GRIA2 inhibited VSMCs behavior, indicating that GRIA2 is a regulator of restenosis. Glutamate receptor 2, encoded by GRIA2, is an important subunit of the AMPA-selective glutamate receptor. 
A
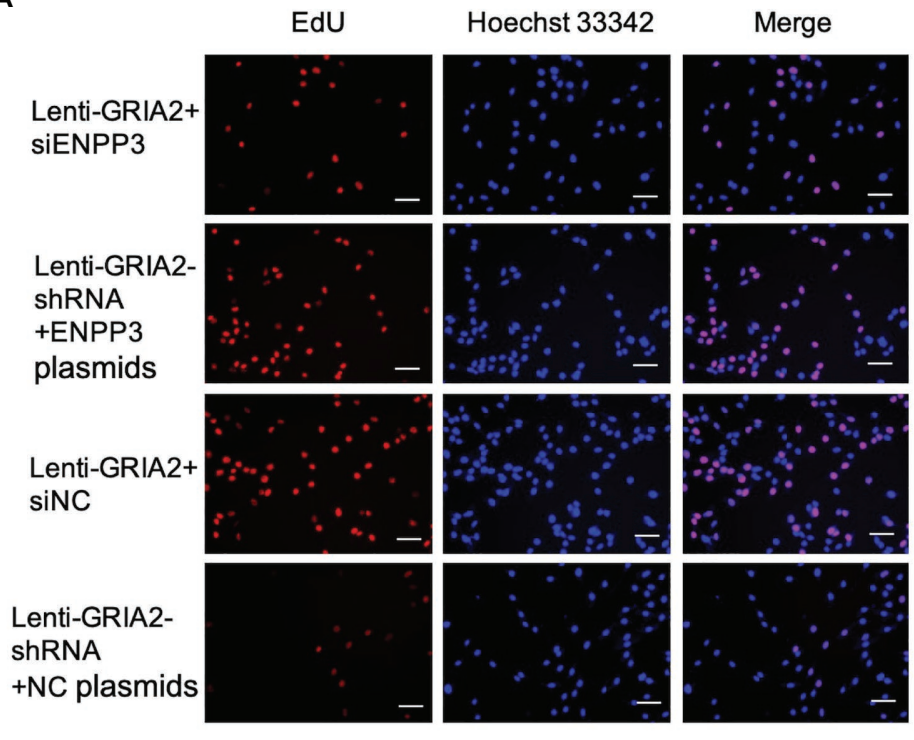

C

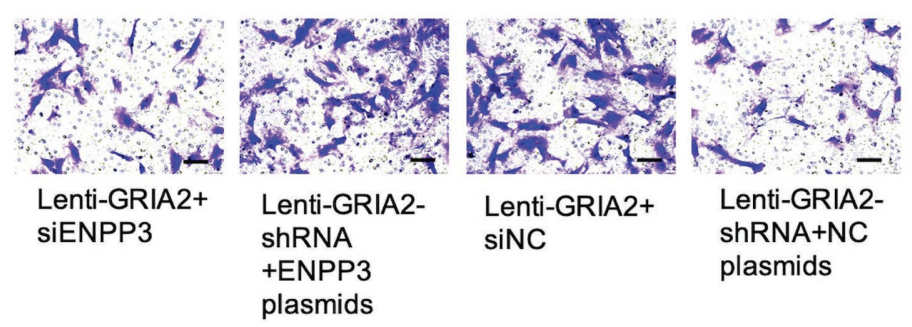

B
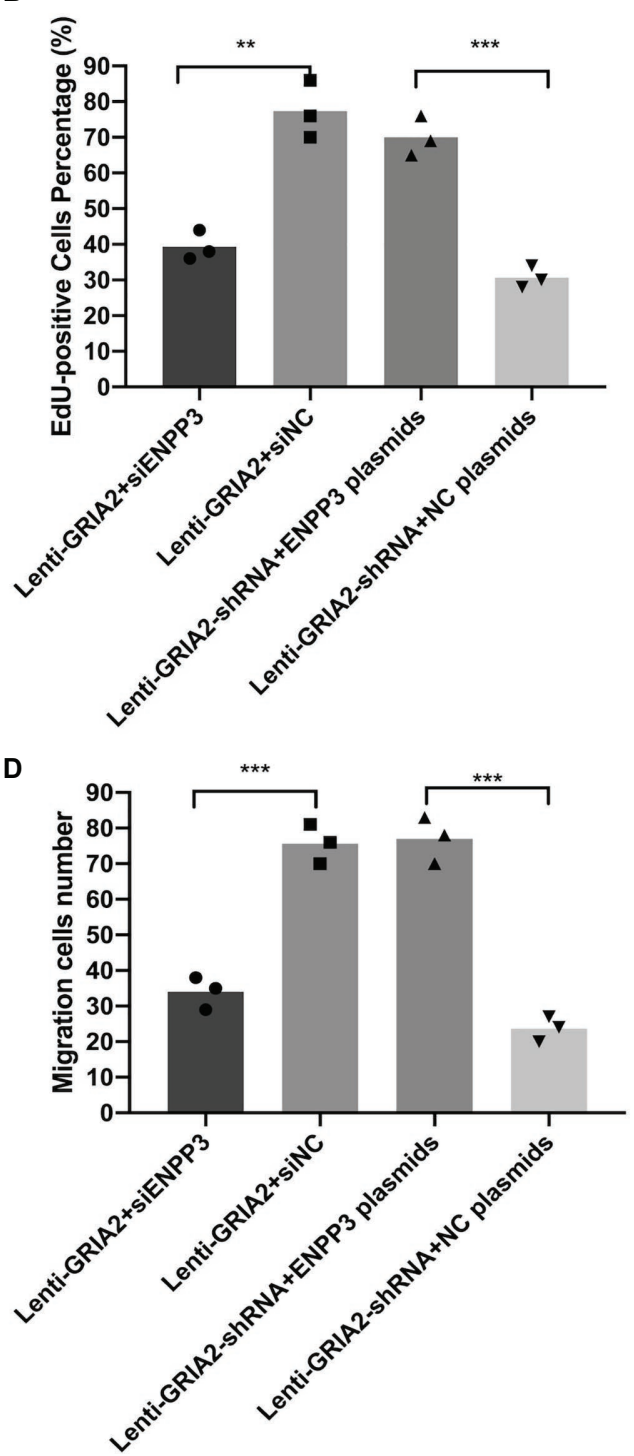

FIGURE 5 | GRIA2 interacted with ENPP3 and regulated the proliferation and migration of VSMCs. (A,B) siENPP3 and ENPP3 plasmids and siNC and shRNA NC plasmids were transfected into GRIA2 lentivirus-transfected VSMCs. The EdU assay illustrated the proliferation of VSMCs (scale bar, $50 \mu m$; $n=3$ ). (C,D) Transwell assays illustrated the migration of VSMCs (scale bar, $100 \mu \mathrm{m} ; n=3$ ). Images are representative, and the bar graph shows the average data of four independent experiments $(n=3)$. Images of EdU and Transwell migration assays were taken at $200 \times$ magnification. Data are presented as the mean \pm SEM. ${ }^{\star *}$ Statistically significant difference $(p<0.01)$ and ${ }^{* \star *}$ statistically significant difference $(p<0.001)$. VSMCs: vascular smooth muscle cells.

Glutamate receptor 2 incorporation is known to greatly reduce the permeability of receptors to $\mathrm{Ca}^{2+}$ and plays an important role in cellular $\mathrm{Ca}^{2+}$ homeostasis (Lee et al., 2010; Liu et al., 2010; Oakes et al., 2017; Tischbein et al., 2019; Konen et al., 2020). Recent evidence has shown that GRIA2 expression levels are linked with VSMC phenotypic switching. Under physiological conditions, VSMCs normally maintain a contractile phenotype. In response to vascular injury, VSMCs undergo phenotypic switching to a dedifferentiated state characterized by excessive proliferation and migration. In addition, studies have also reported that GRIA2 signaling regulates the expression of a gene set involved in the MAPK/ERK signaling pathway
(Gallina et al., 2021). Because the MAPK/ERK pathway plays a crucial role in cell proliferation, differentiation, and migration, the mechanism by which GRIA2 regulates VSMCs proliferation and migration may be through the MAPK/ERK signaling pathway.

To gain more insight into the mechanism of GRIA2 in restenosis, we predicted the downstream target of GRIA2 through the reanalysis of the GSE23314 and GSE53274 datasets. A total of 1852 common DEGs were subjected to KEGG enrichment analysis. The results showed that the DEGs were highly enriched in metabolic pathways. Data on PPIs provide valuable insight into molecular networks. By PPI network analysis, ENPP3, an N-glycosylated protein (Boggavarapu et al., 2016), was predicted 
to be the core protein in restenosis. ENPP3 is a newly described molecule and, therefore, is less studied. The expression of ENPP3 in general has mainly been reported in basophil and mast cells (Buhring et al., 2004; Trapero et al., 2018). Recent studies have shown that ENPP3 is involved in the invasion of tumor cells (Yano et al., 2003, 2004), which is similar to the process of VSMCs migration and proliferation from the media to the intima and forms restenotic plaques. Here, in our study, the expression and localization of ENPP3 were detected. In restenotic plaques, the ENPP3 was also increased and located in VSMCs in the intima, correlated with GRIA2 expression, suggesting that ENPP3 might interact with GRIA2. ENPP3 is a type II transmembrane protein (Buhring et al., 2001) that is involved in hydrolysis of extracellular nucleotides and possesses ATPase and ATP pyrophosphatase activities (Reigada et al., 2005; Levesque et al., 2007). No previous report has demonstrated the expression and interaction between GRIA2 and ENPP3. However, studies have shown that ENPP3 is usually located in neurons (Nishida et al., 2018). Moreover, as ENPP3 is a membrane protein, and GRIA2 possesses a certain degree of cell membrane protein modulation. However, these results indicate that GRIA2 promotes VSMCs effects through ENPP3. To investigate the effect of GRIA2 on ENPP3, we modulated GRIA2. With GRIA2 intervention, the expression of ENPP3 was positively regulated. Our results further showed that GRIA2-promoted VSMCs proliferation and migration were inhibited by ENPP3 repression, implying that GRIA2 interacted with ENPP3 and further regulated VSMCs behavior in restenosis. Further studies are needed to clarify their potential interactions. In present study, we used a double-balloon injury animal model, which consisted of two steps injuries: the first balloon injury was to induce atherosclerosis plaques and, after the plaques were established, the second balloon injury was performed in the same place to mimic the condition of PTA. We found the plaques developed after the two steps injuries are typically hyperplasia foci of VSMCs and extracellular matrix, which is more closely to human restenosis pathology than simple balloon injury protocol (Strauss et al., 1996; Zhou et al., 2016; Zou et al., 2020).

Our results have demonstrated that GRIA2 has a significant impact on restenosis by modulating VSMCs proliferation and

\section{REFERENCES}

Abdul, M., and Hoosein, N. (2005). N-methyl-D-aspartate receptor in human prostate cancer. J. Membr. Biol. 205, 125-128. doi: 10.1007/s00232-005-0777-0

Apelqvist, J., Elgzyri, T., Larsson, J., Londahl, M., Nyberg, P., and Thorne, J. (2011). Factors related to outcome of neuroischemic/ischemic foot ulcer in diabetic patients. J. Vasc. Surg. 53, 1582.e2-1588.e2. doi: 10.1016/j. jvs.2011.02.006

Bennett, M. R., Sinha, S., and Owens, G. K. (2016). Vascular smooth muscle cells in atherosclerosis. Circ. Res. 118, 692-702. doi: 10.1161/CIRCRESAHA.115.306361

Boggavarapu, N. R., Lalitkumar, S., Joshua, V., Kasvandik, S., Salumets, A., Lalitkumar, P. G., et al. (2016). Compartmentalized gene expression profiling of receptive endometrium reveals progesterone regulated ENPP3 is differentially expressed and secreted in glycosylated form. Sci. Rep. 6:33811. doi: 10.1038/ srep33811

Buhring, H. J., Seiffert, M., Giesert, C., Marxer, A., Kanz, L., Valent, P., et al. (2001). The basophil activation marker defined by antibody 97A6 is identical migration. We also explored the mechanism by which upregulated GRIA2 regulates VSMCs behavior as the upstream of ENPP3. Targeting GRIA2-ENPP3 may have potential in the treatment of restenosis post-PTA in lower extremity arteries.

\section{DATA AVAILABILITY STATEMENT}

The datasets presented in this study can be found in online repositories. The names of the repository/repositories and accession number(s) can be found in the article/ supplementary material.

\section{ETHICS STATEMENT}

The animal study was reviewed and approved by the Ethics Committee of Xuanwu Hospital affiliated with Capital Medical University.

\section{AUTHOR CONTRIBUTIONS}

MZ and YG designed the methods and analyzed the data and results. MZ and LQ wrote the manuscript and prepared figures. All authors contributed to the article and approved the submitted version.

\section{FUNDING}

This study was supported by the National Key Research and Development Program of China (no. 2017YFC1104100).

\section{ACKNOWLEDGMENTS}

Our study was supported by the resources and facilities of the core laboratory of Xuanwu Hospital affiliated with Capital Medical University (Beijing, China).

to the ectonucleotide pyrophosphatase/phosphodiesterase 3. Blood 97, 3303-3305. doi: 10.1182/blood. v97.10.3303

Buhring, H. J., Streble, A., and Valent, P. (2004). The basophil-specific ectoenzyme E-NPP3 (CD203c) as a marker for cell activation and allergy diagnosis. Int. Arch. Allergy Immunol. 133, 317-329. doi: 10.1159/000077351

Criqui, M. H., Fronek, A., Barrett-Connor, E., Klauber, M. R., Gabriel, S., and Goodman, D. (1985). The prevalence of peripheral arterial disease in a defined population. Circulation 71, 510-515. doi: 10.1161/01. CIR.71.3.510

Diehm, C., Allenberg, J. R., Pittrow, D., Mahn, M., Tepohl, G., Haberl, R. L., et al. (2009). Mortality and vascular morbidity in older adults with asymptomatic versus symptomatic peripheral artery disease. Circulation 120, 2053-2061. doi: 10.1161/CIRCULATIONAHA.109.865600

Doncheva, N. T., Morris, J. H., Gorodkin, J., and Jensen, L. J. (2019). Cytoscape StringApp: network analysis and visualization of proteomics data. J. Proteome Res. 18, 623-632. doi: 10.1021/acs.jproteome.8b00702

Gallina, A. L., Rykaczewska, U., Wirka, R. C., Caravaca, A. S., Shavva, V. S., Youness, M., et al. (2021). AMPA-type glutamate receptors associated with 
vascular smooth muscle cell subpopulations in atherosclerosis and vascular injury. Front. Cardiovasc. Med. 8:655869. doi: 10.3389/fcvm.2021.655869

Gomez, D., and Owens, G. K. (2012). Smooth muscle cell phenotypic switching in atherosclerosis. Cardiovasc. Res. 95, 156-164. doi: 10.1093/cvr/cvs115

Grunewald, I., Vollbrecht, C., Meinrath, J., Meyer, M. F., Heukamp, L. C., Drebber, U., et al. (2015). Targeted next generation sequencing of parotid gland cancer uncovers genetic heterogeneity. Oncotarget 6, 18224-18237. doi: 10.18632 /oncotarget.4015

Hansen, K. B., Yuan, H., and Traynelis, S. F. (2007). Structural aspects of AMPA receptor activation, desensitization and deactivation. Curr. Opin. Neurobiol. 17, 281-288. doi: 10.1016/j.conb.2007.03.014

Hiatt, W. R., Hoag, S., and Hamman, R. F. (1995). Effect of diagnostic criteria on the prevalence of peripheral arterial disease. The San Luis Valley diabetes study. Circulation 91, 1472-1479. doi: 10.1161/01.CIR.91.5.1472

Ishiuchi, S., Yoshida, Y., Sugawara, K., Aihara, M., Ohtani, T., Watanabe, T., et al. (2007). $\mathrm{Ca}^{2+}$-permeable AMPA receptors regulate growth of human glioblastoma via Akt activation. J. Neurosci. Off. J. Soc. Neurosci. 27, 7987-8001. doi: 10.1523/JNEUROSCI.2180-07.2007

Konen, L. M., Wright, A. L., Royle, G. A., Morris, G. P., Lau, B. K., Seow, P. W., et al. (2020). A new mouse line with reduced GluA2 Q/R site RNA editing exhibits loss of dendritic spines, hippocampal CA1-neuron loss, learning and memory impairments and NMDA receptor-independent seizure vulnerability. Mol. Brain 13:27. doi: 10.1186/s13041-020-0545-1

LeBlanc, V. G., and Marra, M. A. (2015). Next-generation sequencing approaches in cancer: where have they brought us and where will they take us? Cancers 7, 1925-1958. doi: 10.3390/cancers7030869

Lee, S., Yang, G., Yong, Y., Liu, Y., Zhao, L., Xu, J., et al. (2010). ADAR2dependent RNA editing of GluR2 is involved in thiamine deficiency-induced alteration of calcium dynamics. Mol. Neurodegener. 5:54. doi: 10.1186/1750-1326-5-54

Levesque, S. A., Lavoie, E. G., Lecka, J., Bigonnesse, F., and Sevigny, J. (2007). Specificity of the ecto-ATPase inhibitor ARL 67156 on human and mouse ectonucleotidases. Br. J. Pharmacol. 152, 141-150. doi: 10.1038/sj.bjp.0707361

Liistro, F., Angioli, P., Porto, I., Ducci, K., Falsini, G., Ventoruzzo, G., et al. (2019). Drug-eluting balloon versus drug-eluting stent for complex femoropopliteal arterial lesions: the DRASTICO study. J. Am. Coll. Cardiol. 74, 205-215. doi: 10.1016/j.jacc.2019.04.057

Liistro, F., Porto, I., Angioli, P., Grotti, S., Ricci, L., Ducci, K., et al. (2013). Drug-eluting balloon in peripheral intervention for below the knee angioplasty evaluation (DEBATE-BTK): a randomized trial in diabetic patients with critical limb ischemia. Circulation 128, 615-621. doi: 10.1161/ CIRCULATIONAHA.113.001811

Liu, S. Q., and Cull-Candy, S. G. (2000). Synaptic activity at calcium-permeable AMPA receptors induces a switch in receptor subtype. Nature 405, 454-458. doi: $10.1038 / 35013064$

Liu, Y., Formisano, L., Savtchouk, I., Takayasu, Y., Szabo, G., Zukin, R. S., et al. (2010). A single fear-inducing stimulus induces a transcription-dependent switch in synaptic AMPAR phenotype. Nat. Neurosci. 13, 223-231. doi: $10.1038 / \mathrm{nn} .2474$

Louis, S. F., and Zahradka, P. (2010). Vascular smooth muscle cell motility: From migration to invasion. Exp. Clin. Cardiol. 15, e75-e85.

Meves, S. H., Diehm, C., Berger, K., Pittrow, D., Trampisch, H. J., Burghaus, I., et al. (2010). Peripheral arterial disease as an independent predictor for excess stroke morbidity and mortality in primary-care patients: 5-year results of the getABI study. Cerebrovasc. Dis. 29, 546-554. doi: $10.1159 / 000306640$

Nakagawa, T., Cheng, Y., Ramm, E., Sheng, M., and Walz, T. (2005). Structure and different conformational states of native AMPA receptor complexes. Nature 433, 545-549. doi: 10.1038/nature03328

Newby, A. C., and Zaltsman, A. B. (2000). Molecular mechanisms in intimal hyperplasia. J. Pathol. 190, 300-309. doi: 10.1002/(SICI)1096-9896(200002) 190:3<300::AID-PATH596>3.0.CO;2-I

Ni, T., Huang, T., Gu, S. L., Wang, J., Liu, Y., Sun, X., et al. (2020). DRG neurons promote perineural invasion of endometrial cancer via GluR2. J. Cancer 11, 2518-2528. doi: 10.7150/jca.40055

Nishida, K., Nomura, Y., Kawamori, K., Ohishi, A., and Nagasawa, K. (2018). ATP metabolizing enzymes ENPP1, 2 and 3 are localized in sensory neurons of rat dorsal root ganglion. Eur. J. Histochem. 62:2877. doi: 10.4081/ejh. 2018.2877
Oakes, E., Anderson, A., Cohen-Gadol, A., and Hundley, H. A. (2017). Adenosine deaminase that acts on RNA 3 (ADAR3) binding to glutamate receptor subunit B pre-mRNA inhibits RNA editing in glioblastoma. J. Biol. Chem. 292, 4326-4335. doi: 10.1074/jbc.M117.779868

Peterson, S., Hasenbank, M., Silvestro, C., and Raina, S. (2017). IN.PACT ${ }^{\mathrm{TM}}$ admiral $^{\mathrm{TM}}$ drug-coated balloon: durable, consistent and safe treatment for femoropopliteal peripheral artery disease. Adv. Drug Deliv. Rev. 112, 69-77. doi: $10.1016 /$ j. addr. 2016.10 .003

Rabbani, B., Nakaoka, H., Akhondzadeh, S., Tekin, M., and Mahdieh, N. (2016). Next generation sequencing: implications in personalized medicine and pharmacogenomics. Mol. BioSyst. 12, 1818-1830. doi: 10.1039/C6MB00115G

Reigada, D., Lu, W., Zhang, X., Friedman, C., Pendrak, K., McGlinn, A., et al. (2005). Degradation of extracellular ATP by the retinal pigment epithelium. Am. J. Physiol. Cell Physiol. 289, C617-C624. doi: 10.1152/ajpcell.00542.2004

Rzeski, W., Turski, L., and Ikonomidou, C. (2001). Glutamate antagonists limit tumor growth. Proc. Natl. Acad. Sci. U. S. A. 98, 6372-6377. doi: 10.1073/ pnas.091113598

Schmidt, A., Piorkowski, M., Gorner, H., Steiner, S., Bausback, Y., Scheinert, S., et al. (2016). Drug-coated balloons for complex femoropopliteal lesions: 2-year results of a real-world registry. JACC Cardiovasc. Interv. 9, 715-724. doi: $10.1016 /$ j.jcin.2015.12.267

Selvin, E., and Erlinger, T. P. (2004). Prevalence of and risk factors for peripheral arterial disease in the United States: results from the National Health and nutrition examination survey, 1999-2000. Circulation 110, 738-743. doi: 10.1161/01.CIR.0000137913.26087.F0

Song, I., Kamboj, S., Xia, J., Dong, H., Liao, D., and Huganir, R. L. (1998). Interaction of the N-ethylmaleimide-sensitive factor with AMPA receptors. Neuron 21, 393-400. doi: 10.1016/S0896-6273(00)80548-6

Strauss, B. H., Robinson, R., Batchelor, W. B., Chisholm, R. J., Ravi, G., Natarajan, M. K., et al. (1996). In vivo collagen turnover following experimental balloon angioplasty injury and the role of matrix metalloproteinases. Circ. Res. 79, 541-550. doi: 10.1161/01.RES.79.3.541

Takeda, M., Haga, M., Yamada, H., Kinoshita, M., Otsuka, M., Tsuboi, S., et al. (2000). Ionotropic glutamate receptors expressed in human retinoblastoma Y79 cells. Neurosci. Lett. 294, 97-100. doi: 10.1016/ S0304-3940(00)01546-9

Tischbein, M., Baron, D. M., Lin, Y. C., Gall, K. V., Landers, J. E., Fallini, C., et al. (2019). The RNA-binding protein FUS/TLS undergoes calcium-mediated nuclear egress during excitotoxic stress and is required for GRIA2 mRNA processing. J. Biol. Chem. 294, 10194-10210. doi: 10.1074/jbc.RA118.005933

Trapero, C., Jover, L., Fernandez-Montoli, M. E., Garcia-Tejedor, A., Vidal, A., Gomez de Aranda, I., et al. (2018). Analysis of the ectoenzymes ADA, ALP, ENPP1, and ENPP3, in the contents of ovarian endometriomas as candidate biomarkers of endometriosis. Am. J. Reprod. Immunol. 79. doi: 10.1111/aji.12794

Yano, Y., Hayashi, Y., Sano, K., Nagano, H., Nakaji, M., Seo, Y., et al. (2004). Expression and localization of ecto-nucleotide pyrophosphatase/ phosphodiesterase I-1 (E-NPP1/PC-1) and -3 (E-NPP3/CD203c/PD-Ibeta/ B10/gp130(RB13-6)) in inflammatory and neoplastic bile duct diseases. Cancer Lett. 207, 139-147. doi: 10.1016/j.canlet.2003.11.002

Yano, Y., Hayashi, Y., Sano, K., Shinmaru, H., Kuroda, Y., Yokozaki, H., et al. (2003). Expression and localization of ecto-nucleotide pyrophosphatase/ phosphodiesterase I-3 (E-NPP3/CD203c/PD-I beta/B10/gp130RB13-6) in human colon carcinoma. Int. J. Mol. Med. 12, 763-766

Yoshioka, A., Ikegaki, N., Williams, M., and Pleasure, D. (1996). Expression of N-methyl-D-aspartate (NMDA) and non-NMDA glutamate receptor genes in neuroblastoma, medulloblastoma, and other cells lines. J. Neurosci. Res. 46, 164-178. doi: 10.1002/(SICI)1097-4547(19961015)46:2<164::AID-JNR4> 3.0.CO;2-F

Zeller, T., Sixt, S., and Rastan, A. (2009). New techniques for endovascular treatment of peripheral artery disease with focus on chronic critical limb ischemia. Vasa 38, 3-12. doi: 10.1024/0301-1526.38.1.3

Zhang, H. Y., Yang, W., and Lu, J. B. (2017). Knockdown of GluA2 induces apoptosis in non-small-cell lung cancer A549 cells through the p53 signaling pathway. Oncol. Lett. 14, 1005-1010. doi: 10.3892/ol.2017.6234

Zhou, X., Dong, J., Zhang, L., Liu, J., Dong, X., Yang, Q., et al. (2015). Hyperglycemia has no effect on development of restenosis after percutaneous transluminal angioplasty (PTA) in a diabetic rabbit model. J. Endocrinol. 224, 119-125. doi: 10.1530/JOE-14-0391 
Zhou, X., Mou, Y., Shen, X., Yang, T., Liu, J., Liu, F., et al. (2016). The role of atorvastatin on the restenosis process post-PTA in a diabetic rabbit model. BMC Cardiovasc. Disord. 16:153. doi: 10.1186/s12872-0160324-1

Zou, Z., Zhou, X., Zhang, R., Zhang, Q., Jiang, S., Xu, C., et al. (2020). Lin28a up-regulation is associated with the formation of restenosis via promoting proliferation and migration of vascular smooth muscle cells. J. Cell. Mol. Med. 24, 9682-9691. doi: $10.1111 /$ jcmm.15506

Conflict of Interest: The authors declare that the research was conducted in the absence of any commercial or financial relationships that could be constructed as a potential conflict of interest.
Publisher's Note: All claims expressed in this article are solely those of the authors and do not necessarily represent those of their affiliated organizations, or those of the publisher, the editors and the reviewers. Any product that may be evaluated in this article, or claim that may be made by its manufacturer, is not guaranteed or endorsed by the publisher.

Copyright (c) 2021 Zhou, Qi and Gu. This is an open-access article distributed under the terms of the Creative Commons Attribution License (CC BY). The use, distribution or reproduction in other forums is permitted, provided the original author(s) and the copyright owner(s) are credited and that the original publication in this journal is cited, in accordance with accepted academic practice. No use, distribution or reproduction is permitted which does not comply with these terms. 